Comment. Math. Helv. 74 (1999) 419-441

(C) 1999 Birkhäuser Verlag, Basel

\title{
Riemannian submersions of open manifolds which are flat at infinity
}

Valery Marenich

\author{
To my wife
}

\begin{abstract}
We prove that a base $B^{n-k}$ of a Riemannian submersion $\pi: M^{n} \rightarrow B^{n-k}$ is flat, if $M^{n}$ is flat at infinity and $B^{n-k}$ is compact. As a corollary we obtain a topological gapphenomenon for open manifolds of nonnegative sectional curvature (Eschenburg-Schroeder-Strake conjecture).
\end{abstract}

Mathematics Subject Classification (1991). 53C20, 53C21.

Keywords. Open manifold, riemannian submersion.

\section{Introduction}

We say that an open (complete noncompact) Riemannian manifold $M^{n}$ is flat at infinity if its sectional curvatures $K_{\sigma}$ at a point tend to zero as this point tends to infinity. More precisely, let $o$ be some fixed point in $M^{n}$ and

$$
\varkappa(\rho)=\sup \left\{\left|K_{\sigma}\right| \mid \sigma \subset T_{q} M^{n}, \rho(o, q)=\rho\right\}
$$

be the function which measures the absolute value of the curvature of $M^{n}$ as $\rho \rightarrow \infty$. Then $M^{n}$ is flat at infinity if $\varkappa(\rho) \rightarrow 0$ as $\rho \rightarrow \infty$.

Riemannian submersion $\pi: M^{n} \rightarrow B^{n-k}$, is a map of constant rank such that:

1. On $M^{n}$ is defined a smooth horizontal distribution $\mathcal{H}$ of subspaces which are orthogonal to the distribution $\mathcal{V}$ of subspaces tangent to fibers $W_{p}=\pi^{-1}(b), b \in$ $B^{n-k}$, which we call vertical ones.

2. In every point $q$ the restriction of the differential $\pi_{*}$ of the map $\pi$ to a horizontal space $\mathcal{H}_{q}$ is an isometry.

A union of vertical fibers of a Riemannian submersion gives a metric foliation on $M^{n}$, i.e., a smooth partition into lower dimensional submanifolds which are locally everywhere equidistant, see [GG].

The purpose of this paper is to prove the following result. 
Theorem A. If the space $M^{n}$ of a Riemannian submersion $\pi: M^{n} \rightarrow B^{n-k}$ is flat at infinity, and the base $B^{n-k}$ is compact, then the manifold $B^{n-k}$ is flat.

We would like to stress, that in Theorem A we do not assume any restriction on the sign of the curvature or any estimate on the injectivity radius. In fact, as follows from the proof it is sufficient to suppose that only sectional curvatures in two-dimensional directions containing some horizontal vector tend to zero at infinity. It is also easy to see that our Theorem A does not include unnecessary conditions.

According to [CG] every open Riemannian manifold $V^{n}$ of nonnegative sectional curvature is diffeomorphic to the space of a normal bundle of some closed totally geodesic submanifold $S$ of $V^{n}$ ( $S$ is called a soul of $\left.V^{n}\right)$, and due to [P] there exists a Riemannian submersion $\pi: V^{n} \rightarrow S$. Therefore, Theorem A yields the following corollary for manifolds of nonnegative sectional curvature.

Theorem B (Eschenburg-Schroeder-Strake conjecture). If an open Riemannian manifold $V^{n}$ of nonnegative sectional curvature is flat at infinity, then the soul $S$ of this manifold is flat, and the universal cover $\widetilde{V}^{n}$ of $V^{n}$ is diffeomorphic to the Euclidean space $R^{n}$.

The last theorem means that if $V^{n}$ is simply connected and $\varkappa(\rho) \rightarrow 0$ as $\rho \rightarrow \infty$, then $V$ is diffeomorphic to the euclidean space; i.e., Theorem B may be considered as the topological counterpart ("topological gap-phenomenon") of a well known metric gap-phenomenon asserting that if the manifold $V^{n}$ has a pole, sectional curvature of $V^{n}$ is non-negative or non-positive and $\rho^{2} \varkappa(\rho) \rightarrow 0$ as $\rho \rightarrow \infty$, then $V^{n}$ is isometric to the euclidean space $R^{n}$, see [KS]. Theorem B was conjectured in [ESS] and proved there for the case codim $S \leq 3$ (see also announcement in [M1]).

The proof of Theorem A is based on a uniform length estimate for some special curves in Grassmanians and ergodic arguments first introduced in [M2] (see also [M1, M3]). Here we present shortly the idea of the proof. Taking a long geodesic $\gamma(t)$ on the base $B^{n-k}$ and some parallel vector field $\bar{Y}(t)$ along $\gamma$ we construct their horizontal lifts sufficiently close to infinity in $M^{n}$, i.e., a geodesic $l(t)$ and a horizontal vector field $Y(t)$ along $l$ such that the curvature of $M^{n}$ along $l$ is small. Due to the O'Neill fundamental equation for submersions we know that

$$
\bar{K}_{B}(\gamma(t), \dot{\gamma}(t), \bar{Y}(t))=K_{M}(l(t), \dot{l}(t), Y(t))+3\left\|A_{i(t)}(Y)(t)\right\|^{2},
$$

where $\bar{K}_{B}$ and $K_{M}$ are sectional curvatures of the base $B^{n-k}$ and $M^{n}$ correspondingly and $A$ is the O'Neill fundamental tensor of submersion, see [O'N1]. Then we note, that

$$
\left\|A_{i(t)}(Y)(t)\right\| \leq\left\|\dot{V}_{l}(t)\right\|,
$$

where $V_{l}(t)$ is a curve in a Grassmann manifold $G_{n}^{k}$ of $k$-dimensional subspaces consisting of parallel transports of vertical subspaces of a submersion along $l$ to 
some fixed point. Because subspaces $V_{l}(t)$ are generated by Jacobi fields along $l$ where the curvature of $M^{n}$ is small, we see that every such curve tends in $C^{1}$-norm to a curve in $G_{n}^{k}$ of some special kind - we call such curves linear curves in $G_{n}^{k}$. We prove that length of linear curves are uniformly bounded (our main technical result, see Lemma 3 ), and that compactness of the base $B^{n-k}$ of the submersion yields a uniform boundedness of $\left\|\dot{V}_{l}(0)\right\|$. This leads to our principal estimates:

$$
\int_{0}^{T}\left\|\dot{V}_{l}(t)\right\| d t \quad \text { and } \quad \int_{0}^{T}\left\|A_{i(t)}(Y)(t)\right\|^{2} d t
$$

are uniformly bounded for some $T$ depending on $l$ and tending to infinity as $l$ tends to infinity in $M^{n}$, see Lemma 7. Due to O'Neill formula this implies that ergodic means of the sectional curvature of the base tend to zero:

$$
\frac{1}{T} \int_{0}^{T}\left|\bar{K}_{B}(\gamma(t), \dot{\gamma}(t), \bar{Y}(t))\right| d t \rightarrow 0,
$$

which due to Birkhoff-Khintchin theorem yields the vanishing of the sectional curvature, i.e., proving that the base $B^{n-k}$ is flat and completing the proof of Theorem A.

A formula (8.1) below for the derivative of the trace $A_{1}(t)$ of second forms $\mathbf{I I}(t)$ of fibers along a horizontal geodesic $l(t)$ in $M^{n}$ from [M2], [M3]

$$
A_{1}^{\prime}(t)+\left\|A_{2}(t)\right\|=\left\|\dot{V}_{l}(t)\right\|^{2}+K_{M}^{k}(l(t), i(t))
$$

where $A_{2}(t)$ denotes the sum of squares of eigenvalues of $\mathbf{I I}(t)$ and $K_{M}^{k}(l(t), \dot{l}(t))$ - "partial (or vertical)" Ricci curvature of $M^{n}$, implies in addition that normal curvatures of fibers tend to zero at infinity.

Another corollary of our Theorem A is the following already known result (see $[\mathrm{W}])$.

Theorem C. All flat spaces of riemannian submersions over compact bases are locally direct products.

Indeed, due to O'Neill this statement is true if all fibers of the submersion $\pi: M^{n} \rightarrow B^{n-k}$ are totally geodesic ones, and O'Neill's fundamental tensor $A$ of the submersion $\pi$ is zero. To prove this when $M^{n}$ is flat it is sufficient to apply our ergodic arguments to the last equality. The reader may find these arguments in [M2, M3].

The organization of the paper is as follows. First we consider linear curves in Grassmanians. We prove that length of such curves are uniformly bounded by some constant. Then we define curves of vertical subspaces for a submersion $\pi: M^{n} \rightarrow B^{n-k}$, prove that these curves converge in $C^{1}$-norm to linear curves and obtain our principal estimates. To complete the proof of Theorem A we use ergodic arguments from our previous articles [M1 - M3].

The author express his sincere gratitude to the referee for the extraordinarily careful reading and very useful criticism. 


\section{Linear curves of subspaces}

Denote by $G_{n}^{k}$ the Grassmann manifold of all $k$-dimensional subspaces of the euclidean $n$-dimensional space $R^{n}$. $G_{n}^{k}$ is considered with a natural Riemannian metric $g$ such that the factorization $\Pi: O(n) \rightarrow O(n) / O(k) \times O(n-k)=G_{n}^{k}$, where $O(n)$ is provided with the Lipcshitz-Killing metric, is a riemannian submersion.

We say that a curve $V(t), 0 \leq t \leq T$ in $G_{n}^{k}$ of $k$-subspaces is linear if the subspaces $V(t)$ are generated by some vectors $\eta_{i}(t), i=1, \ldots, k$ linearly depending on $t$, i.e., such that

$$
\eta_{i}(t)=\eta_{i}(0)+t D \eta_{i}(0) .
$$

Vectors $\eta_{i}(t)$ are defined for all $t$, but the vector space generated by them may have dimension less than $k$ for some values of the parameter $t$. If it is not stated otherwise, we assume that $[0, T)$ is the maximal interval where this vector space has constant dimension $k$. Obviously, the choice of a linear base (1.1) of $V(t)$ is not unique. Among different possibilities we always can choose some orthonormal base $\left\{e_{1}, \ldots, e_{k}, e_{k+1}, \ldots, e_{n}\right\}$ of $R^{n}$ such that vectors $\left\{e_{1}, \ldots, e_{k}\right\}$ generate $V(0)$ and $V(t)$ is generated by $\left\{e_{1}+t d_{1}, \ldots, e_{k}+t d_{k}\right\}$ for some vectors $d_{i}=d_{i}^{j} e_{j}$ which we call derivatives of $e_{i}$. Continuing by linearity the map $e_{i} \rightarrow d_{i}$ we define some linear map $A: V(0) \rightarrow R^{n}$.

In Lemmas 1 and 2 below we assume one more condition on $V(t)$. Further (see section 2) we consider the curve $V_{l}(t)$ of vector spaces which are parallel transports along some horizontal geodesic $l(t)$ of vertical subspaces of a submersion $\pi$ to a fixed point $q=l(0)$. Thus $V_{l}(t)$ is generated by all horizontal variations of $l$, i.e., by some Jacobi fields $\eta_{i}(t)$ along $l$. In this case vectors $D \eta_{i}(0)$ are covariant derivatives of these Jacobi fields, and for two arbitrary vectors $v$ and $w$ of $V_{l}(0)$ the bilinear form $(A v, w)$ is the second fundamental form of the fiber corresponding to the normal $i(0)$, see section 2 below. Because of this below in Lemmas 1 and 2 the form $(A v, w)$ is symmetric at $t=0$. Therefore, we can choose the base $\left\{e_{i}, i=1, \ldots k\right\}$ of $V(0)$ consisting of eigenvectors of this form. Thus, the tangent to $V(0)$ component of the derivative $d_{i}$ of the base vector $e_{i}$ is parallel to it and

$$
e_{i}(t)=\left(1+t \lambda_{i}\right) e_{i}+t \sum_{j=k+1, \ldots, n}\left(d_{i}\right)^{j} e_{j},
$$

where $\lambda_{i}$ are eigenvalues of the form $(A v, w)$. We say that the linear curve $V(t)$ is determined by $\left\{e_{i}, d_{i} ; i=1, \ldots k\right\}$.

Call vectors of $V(0)$ vertical and normal to $V(0)$ - horizontal ones. Summing up what we said above we see that every linear curve $V(t)$ is the image of the subspace generated by first $k$ coordinate vectors of some orthonormal base $\left\{e_{1}, \ldots, e_{k}, e_{k+1}, \ldots, e_{n}\right\}$ in $R^{n}$ under the map with the following matrix:

$$
\left(\begin{array}{cc}
I+t(\mathcal{V} D) & t(\mathcal{H} D) \\
0 & I
\end{array}\right)
$$


where $\mathcal{V} D$ and $\mathcal{H} D$ are matrices of vertical and horizontal components of the derivatives $d_{i}$ correspondingly: $\mathcal{V} D=\left(d_{i}\right)^{j}, i=1, \ldots, k ; j=1, \ldots, k$ and $\mathcal{H} D=$ $\left(d_{i}\right)^{j}, i=1, \ldots, k ; j=k+1, \ldots, n$, and the matrix $\mathcal{V} D$ is diagonal. To define an orthogonal transformation of $R^{n}$ mapping $V(0)$ into $V(t)$ we use an orthogonalization process as follows. Denote by $\Lambda_{1}^{1}=\left(2 \max \left\{\lambda_{i} \mid i=1, \ldots, k\right\}\right)^{-1}$. Then for $t<\Lambda_{1}^{1}$ the vectors

$$
\tilde{e}_{i}(t)=e_{i}(t) /\left(1+\lambda_{i} t\right)=e_{i}+\left(1+\lambda_{i} t\right)^{-1} t \sum_{j=k+1, \ldots, n}\left(d_{i}\right)^{j} e_{j}, j=k+1, \ldots, n
$$

also generate $V(t)$. If $j=k+1, \ldots, n$ we set

$$
\tilde{e}_{j}(t)=e_{j}-t \sum_{i=1}^{k}\left(d_{i}\right)^{j} e_{i} .
$$

An easy calculation shows

$$
\left|\left(\tilde{e}_{i}(t), \tilde{e}_{j}(t)\right)-\delta_{i j}\right| \leq\left(1+t^{2} \Lambda_{1}^{2}\right) t^{2}\|\mathcal{H} D\|^{2},
$$

where

$$
\|\mathcal{H} D\|^{2}=\sum_{i=1, \ldots, k ; j=k+1, \ldots, n}\left(\left(d_{i}\right)^{j}\right)^{2}
$$

and $\Lambda_{1}^{2}$ some constant depending only on $\Lambda_{1}^{1}$ and dimensions of considered spaces. Because due to (1.4) the base $\tilde{e}_{i}, i=1, \ldots, n$ is already almost orthonormal, another easy calculation shows that for the orthonormal base $\left\{\bar{e}_{i}(t), i=1, \ldots n\right\}$ of $R^{n}$ which is obtained by an orthogonalization from $\left\{\tilde{e}_{i}(t), i=1, \ldots n\right\}$ we have

$$
\left\|\tilde{e}_{i}(t)-\bar{e}_{i}(t)\right\| \leq\left(1+t^{2} \Lambda_{1}^{3}\right) t^{2}\|\mathcal{H} D\|^{2}
$$

where the constant $\Lambda_{1}^{3}$ again depends only on $\Lambda_{1}^{2}$ and dimension $n$. By construction $\left\{\bar{e}_{i}(t), i=1, \ldots n\right\}$ is the orthonormal base of $R^{n}$ such that the first $k$ vectors generate $V(t)$, or $V(t)$ is the image of $V(0)$ under the orthogonal transformation with a matrix:

$$
O(t)=\left(\begin{array}{cc}
I & t(\mathcal{H} D) \\
-t(\mathcal{H} D) & I
\end{array}\right)+t^{2} G
$$

where for the norm of the matrix $G$ the following is true:

$$
\|G\| \leq\left(1+t^{2} \Lambda_{1}^{4}\right)\|\mathcal{H} D\|^{2},
$$

for some constant $\Lambda_{1}^{4}$ depending on $\Lambda_{1}^{3}$. Finally note that the curve $V(t)$ in $G_{n}^{k}$ is the image under submersion $\Pi: O(n) \rightarrow G_{n}^{k}$ of the curve $O(t)$ in $O(n)$ so that the vector $\dot{V}(0)$ is the image of $\dot{O}(0)$ under the differential $\Pi_{*}$. From (1.7) we see 
that the tangent vector of the curve of the orthogonal transformations $O(t)$ at the moment $t=0$ is

$$
\dot{O}(0)=\left(\begin{array}{cc}
0 & \mathcal{H} D \\
-\mathcal{H} D & 0
\end{array}\right)
$$

By definition of the Lipschitz-Killing metric the length of this vector equals $\|\mathcal{H} D\|$. Note also that this vector is the horizontal one for the riemannian submersion $\Pi: O(n) \rightarrow O(n) / O(k) \times O(n-k)=G_{n}^{k}$, so that it has the same length as its image $\dot{V}(0)$ under the differential $\Pi_{*}$. Therefore, the length of the vector $\dot{V}(0)$ in $G_{n}^{k}$ equals the length of $\dot{O}(0)$ in the Lipschitz-Killing metric on $O(n)$, i.e., is exactly $\|\mathcal{H} D\|$; and we arrive at the following statement.

\section{Lemma 1.}

$$
\|\dot{V}(0)\|^{2}=\|\mathcal{H} D\|^{2}=\sum_{i=1, \ldots, k ; j=k+1, \ldots, n}\left(\left(d_{i}\right)^{j}\right)^{2}
$$

Remark 1. Note that if we consider another curve $\tilde{V}(t)$ of $k$-subspaces of $R^{n}$ generated by some other fields $\left\{\mu_{i}(t), i=1, \ldots, k\right\}$ such that $\mu_{i}(0)=\eta_{i}(0)$ and $\left(d \mu_{i}(t) / d t\right)_{\mid t=0}=D \eta_{i}(0)$ in (1.1), but dependence of $\mu_{i}(t)$ on $t$ is not linear, then still $\tilde{V}(0)=\dot{V}(0)$, so that the speed of the curve $V(0)$ depends only on the initial values $\eta_{i}(0)$ and $D \eta_{i}(0)$. Thus, the formula of Lemma 1 is true for an arbitrary curve of $k$-subspaces in $G_{n}^{k}$.

Now we show that a geodesic curvature $K_{g}(V(t))$ of the linear curve $V(t)$ determined by $\left\{e_{i}, d_{i} ; i=1, \ldots k\right\}$ can be estimated at initial point $t=0$ in terms of $\left\{e_{i}, d_{i} ; i=1, \ldots k\right\}$.

Lemma 2. Let $V(t)$ be the linear curve determined by $\left\{e_{i}, d_{i} ; i=1, \ldots k\right\}$, where $\left\{e_{i} ; i=1, \ldots k\right\}$ is the orthonormal base of $V(0)$. Then for some constant $\Lambda_{2}$ depending only on dimension $n$

$$
\left|K_{g}(V(0))\right| \leq \Lambda_{2}
$$

where $K_{g}(V(t))$ is the geodesic curvature of $V(t)$ at the moment $t$.

Proof. To prove this we use a well-known equality between a geodesic curvature of some curve in a Riemannian manifold and a distance from this curve to the geodesic, issuing from the same initial point with the same velocity vector: the geodesic curvature $K_{g}(V(0))$ of the curve $V(t)$ and the norm of its vector of velocity are related by the following equality:

$$
K_{g}(V(0))=\lim _{t \rightarrow 0} 2 \frac{\operatorname{dist}_{G_{n}^{k}}(V(t), \bar{V}(t))}{\|\dot{V}(0)\|^{2} t^{2}},
$$


where $\bar{V}(t)$ is the geodesic in $G_{n}^{k}$ issuing from $V(0)$ with the same velocity vector: $\bar{V}(0)=\dot{V}(0)$. Every geodesic $\bar{V}(t)$ in the base $G_{n}^{k}$ of the riemannian submersion $\Pi: O(n) \rightarrow O(n) / O(k) \times O(n-k)=G_{n}^{k}$ is the image under the map $\Pi$ of some horizontal geodesic in $O(n)$, i.e., some 1-parameter subgroup of orthogonal transformations of $R^{n}$. Consider the 1-parameter subgroup $\bar{O}(t)$ of $O(n)$ generated by the vector $\dot{O}(0)$ above:

$$
\dot{O}(0)=\left(\begin{array}{cc}
0 & \mathcal{H} D \\
-\mathcal{H} D & 0
\end{array}\right)
$$

Because the vector $\dot{O}(0)$ is horizontal the geodesic $\bar{O}(t)$ in $O(n)$ is a horizontal geodesic and goes under submersion $\Pi$ onto some geodesic in $G_{n}^{k}$. Because, as we verified above, the vector $\dot{O}(0)$ has the image under the differential $\Pi_{*}$ which is equal to $\dot{V}(0)$, this geodesic has the same velocity vector at initial point when $t=0$, i.e., we conclude $\bar{V}(t)=\Pi(\bar{O}(t))$. A direct calculation shows that

$$
\bar{O}(t)=\exp (t \dot{O}(0))=I+t \dot{O}(0)+t^{2} \bar{G}+\ldots
$$

where for some constant $\Lambda_{2}^{1}$ we have $\|\bar{G}\| \leq \Lambda_{2}^{1}\|\mathcal{H} D\|^{2}$. Because of the estimates (1.7)-(1.8) above this gives inequality

$$
\|\bar{O}(t)-O(t)\| \leq t^{2} \Lambda_{2}\|\mathcal{H} D\|^{2} / 2
$$

for some constant $\Lambda_{2}$ depending on $\Lambda_{2}^{1}$ and dimension $n$. Because (as every riemannian submersion) $\Pi$ does not increase distances the last formula yields an inequality

$$
\|\bar{V}(t)-V(t)\| \leq t^{2} \Lambda_{2}\|\mathcal{H} D\|^{2} / 2
$$

implying the claim of the lemma. Lemma 2 is proved.

Remark 2. Obviously, as in Remark 1 above, the geodesic curvature of the curve $V(t)$ at $t=0$ depends only on the initial values of the fields, generating $V(t)$ and their first and second derivatives at $t=0$. If some other curve $\tilde{V}(t)$ of $k$ subspaces of $R^{n}$ is generated by some fields $\left\{\mu_{i}(t), i=1, \ldots, k\right\}$ such that $\mu_{i}(0)=$ $\eta_{i}(0),\left(d \mu_{i}(t) / d t\right)_{\mid t=0}=D \eta_{i}(0)$, and $\left\|\left(d^{2} \mu_{i}(t) / d t^{2}\right)_{\mid t=0}\right\| \leq \Lambda_{2}^{2}\|\dot{V}(0)\|^{2}$, then its curvature at $t=0$ is bounded by some constant $\Lambda_{2}^{3}(C)$ depending on $K_{g}(V(0))$ and the constant $\Lambda_{2}^{2}$.

Next Lemma is the main technical point of our arguments.

Lemma 3. Let $V(t), 0 \leq t \leq T$ be some linear curve in $G_{n}^{k}$ such that its geodesic curvature $K_{g}(V(t))$ is bounded by some constant $\Lambda_{2}$ for all $0 \leq t \leq T$. Than the length of $V(t)$ is bounded by some constant $\Lambda_{3}$ which depends on $\Lambda_{2}$, but does not depend on $T$. 
Proof. Proof of the lemma follows from simple compactness arguments. In addition to the Riemannian metric $g$ on $G_{n}^{k}$ defined above and coming from the Lipschitz Killing metric on $O(n)$ under the submersion $\Pi$, the grassmanian $G_{n}^{k}$ admits also the following non-riemannian metric $\angle$ ("angle"): for two subspaces $V$ and $W$ in $R^{n}$ the angle between them is

$$
\angle(V, W)=\sup _{v \in V} \inf _{w \in W} \angle(v, w) .
$$

Clearly, we always have $0 \leq \angle(V, W) \leq \pi / 2$ and $\angle(V, W)=\pi / 2$ if and only if some vector $w$ of $W$ is orthogonal to $V$. Let $V(t)$ be our linear curve determined by $\left\{e_{i}, d_{i} ; i=1, \ldots, k\right\}$ as in (1.2), and also, as before, $A: V(0) \rightarrow R^{n}$ be the linear map continuing by linearity the map $e_{i} \rightarrow d_{i}$. Continue arbitrarily this map to some linear map $\tilde{A}: R^{n} \rightarrow R^{n}$ in a self-adjoint way (i.e., take an arbitrary extension $A^{\prime}$ and then change to $\left(A^{\prime}+\left(A^{\prime}\right)^{*}\right) / 2$ ). Then the space $V(t)$ is the image of $V(0)$ under the map $I+t \tilde{A}$, and for some vector $\eta(0)$ of $V(0)$ we have $(\eta(0), \eta(t))=0$ for $\eta(t)=(I+t \tilde{A}) \eta(0)$ from $V(t)$ if and only if

$$
(\eta(0),(I+t \tilde{A}) \eta(0))=0
$$

Denote by $\left\{E_{i}, i=1, \ldots, n\right\}$ the orthonormal base of $R^{n}$ consisting of eigenvectors of $\tilde{A}$ and by $\lambda_{i}, i=1, \ldots, n$ corresponding eigenvalues. If we take a partition $-\infty<t_{1}<\ldots<t_{m}<\infty$ for $t_{i}=-\left(\lambda_{i}\right)^{-1}, i=1, \ldots, m$, where $\lambda_{i}, i=1, \ldots, m$ are all nonzero eigenvalues, then easy to see, that for arbitrary $t_{i}<t^{\prime}<t^{\prime \prime}<t_{i+1}$ we have

$$
\left(\eta\left(t^{\prime}\right), \eta\left(t^{\prime \prime}\right)\right)>0
$$

where $\eta(t)=(I+t \tilde{A}) \eta(0)$. Last condition implies that the angle between some fixed vector $\eta\left(t^{\prime}\right)$ and $\eta\left(t^{\prime \prime}\right)$ is a monotonely increasing function on $t^{\prime \prime}$ when $t^{\prime}<t^{\prime \prime}$ are from $\left(t_{i}, t_{i+1}\right)$. The same condition implies, that the angle between $\eta\left(t^{\prime \prime}\right)$ and $V\left(t^{\prime}\right)$ is also a monotonely increasing function on $t^{\prime \prime}$ under the same restriction. Indeed, by definition for all $t$ we have $\eta(t)=\eta(0)+t d$ for some $\eta(0)$ from $V(0)$. Let $d=v+w$, where $v$ belongs to the subspace $V\left(t^{\prime}\right)$, and $w$ is normal to it. Let also $\eta\left(t^{\prime}\right)=a+b$, where the vector $a=\lambda v$ is parallel to $v$, and $b$ is normal to $v$. Then $\eta\left(t^{\prime \prime}\right)=b+\left(\lambda+\left(t^{\prime \prime}-t^{\prime}\right)\right) v+\left(t^{\prime \prime}-t^{\prime}\right) w$, and (3.3) means that

$$
\left(\eta\left(t^{\prime}\right), \eta\left(t^{\prime}\right)\right)+\left(t^{\prime \prime}-t^{\prime}\right)\left(\eta\left(t^{\prime}\right), v\right)>0
$$

The component of $\eta\left(t^{\prime \prime}\right)$ normal to $V\left(t^{\prime}\right)$ equals $\left(t^{\prime \prime}-t^{\prime}\right) w$ while the tangent component of $\eta\left(t^{\prime \prime}\right)$ to $V\left(t^{\prime}\right)$ is $\eta\left(t^{\prime}\right)+\left(t^{\prime \prime}-t^{\prime}\right) v$. So for the angle $\phi\left(t^{\prime \prime}\right)$ between $\eta\left(t^{\prime \prime}\right)$ and the subspace $V\left(t^{\prime}\right)$ we have

$$
\operatorname{tg}\left(\phi\left(t^{\prime \prime}\right)\right)=\frac{\sqrt{(w, w)}\left(t^{\prime \prime}-t^{\prime}\right)}{\sqrt{\left(\eta\left(t^{\prime}\right)+\left(t^{\prime \prime}-t^{\prime}\right) v, \eta\left(t^{\prime}\right)+\left(t^{\prime \prime}-t^{\prime}\right) v\right)}}
$$


and by a direct calculation we see, that due to (3.4) the derivative of $\phi(t$ ") is positive. Because this is true for an arbitrary $\eta(t$ ") we conclude that the angle between $V(t ")$ and $V\left(t^{\prime}\right)$ is also a monotonely increasing function on $t$ " when $t^{\prime}<t^{\prime \prime}$ are from $\left(t_{i}, t_{i+1}\right)$. To prove this it is sufficient to note that the derivative of the angle between $V\left(t^{\prime}\right)$ and $V\left(t^{\prime \prime}\right)$ on $t^{\prime \prime}$ equals the maximum of derivatives of angles between $V\left(t^{\prime}\right)$ and those vectors $\eta\left(t^{\prime \prime}\right)$ of $V\left(t^{\prime \prime}\right)$ which have a maximum angle with $V\left(t^{\prime}\right)$.

Thus, every linear curve $V(t)$ we can divide in not more than $n+1$ intervals $\left\{V(t) \mid t_{i}<t<t_{i+1}\right\}$ such that the "angle"-function $\phi_{s}(t)=\angle(V(s), V(s+t))$ is monotonely increasing for $0<t<t_{i+1}-s$ and monotonely decreasing for $t_{i}-s<t<0$; and the claim of the lemma will follow if we prove it for every such interval. In order to do this we note that this monotonicity of the angle means that the curve $\left\{V(t) \mid t_{i}<t<t_{i+1}\right\}$, leaving at some moment $\epsilon$-neighborhood of $V(s)$ in the metric $\angle$ never come back, or that every ball in $\angle$-metric with the center $V(s)$ contains only one connected arc of the considered interval of the curve $V(t)$. Denote by $U(V, \epsilon)$ an $\epsilon$-neighborhood in the metric $\angle$ of the point $V$ of $G_{n}^{k}$.

For a given number $\Lambda_{2}$ there exists some $\omega$ depending on $\Lambda_{2}$ such that in every $\omega$-ball in the Riemannian metric $g$ a length of every connected arc of an arbitrary curve with geodesic curvature bounded by $\Lambda_{2}$ is bounded by some constant $L$, which depends on $\omega$ and $\Lambda_{2}$ and has order $2 \omega$ as $\omega \rightarrow 0$.

Because topologies generated by two metrics $\angle$ and $g$ coincide, there exists a function $\omega(\epsilon)$ (where $\omega(\epsilon) \rightarrow 0$ as $\epsilon \rightarrow 0$ ) such that every $\epsilon$-ball in the metric $\angle$ is contained in $\omega(\epsilon)$-ball in the metric $g$. Find some $\epsilon$ such that $\omega(\epsilon) \leq \omega$. Using compactness of $G_{n}^{k}$ find some finite covering $G_{n}^{k}=\cup_{i} U\left(V_{i}, \epsilon / 2\right)$. If some point $V\left(t^{\prime}\right)$ belongs to some $U\left(V_{i}, \epsilon / 2\right)$, then because of the triangle inequality the intersection of the considered interval $V(t), t_{i}<t<t_{i+1}$ with this $U\left(V_{i}, \epsilon / 2\right)$ lies in $U\left(V\left(t^{\prime}\right), \epsilon\right)$, and by the arguments above has length less than $L$, which obviously means that the length of the whole interval is bounded by $L N$, where $N$ is the number of all $U\left(V_{i}, \epsilon / 2\right)$ in the finite covering of $G_{n}^{k}$. As was said above, the number of intervals $V(t), t_{i}<t<t_{i+1}$ with described behavior of the "angle"-function $\phi_{s}(t)$ is not bigger than the dimension $n$, so that the length of $V(t),-\infty<t<\infty$ is bounded by $n L N$, i.e.,

$$
\int_{-\infty}^{\infty}\|\dot{V}(t)\| d t<\Lambda_{3}
$$

for some constant $\Lambda_{3}$ depending only on $\Lambda_{2}$. Lemma 3 is proved.

Remark 3. Note that the length estimate of the last Lemma is easily generalized for arbitrary family of subspaces $V(t)$ generated by some $k$-vectors linearly depending on $t$, even if for some parameters $\operatorname{dim} V(t) \neq k$, or $V(t) \notin G_{n}^{k}$. Indeed, let $V(t)$ be generated by $\eta_{i}(t)=\eta_{i}(0)+t d_{i}, i=1, \ldots, k$. Then $\operatorname{dim} V(t) \neq k$ if and only if $\left\|\eta_{1}(t) \wedge \ldots \wedge \eta_{k}(t)\right\|=0$. The last function being polynomial of degree $k$ has at most $k$ zeros $t_{i}, i=1, \ldots k^{\prime}, k^{\prime} \leq k$, so that $V_{i}(t)=\left\{V(t), t_{i}<t<t_{i+1}\right\}$ is a linear curve in $G_{n}^{k}$. Therefore, if geodesic curvatures of $V_{i}$ are bounded by $\Lambda_{2}$ 
the sum of length of all curves $V_{i}(t)=\left\{V(t) \mid t_{i}<t<t_{i+1}\right\}$, which we denote by $L(V)$, is not bigger than $(k+1) \Lambda_{3}$ :

$$
\int_{-\infty}^{\infty}\|\dot{V}(t)\| d t \leq(k+1) \Lambda_{3}
$$

where $\|\dot{V}(t)\|$ is given by the formula of Lemma 1 .

This remark will be essential in the next section when we shall consider $V(t)$ defined as above (i.e., generated by vectors linearly depending on $t$ ) and such that probably $\operatorname{dim} V(t) \not \equiv k$, i.e., may be $\operatorname{dim} V(t)<k$ for some $t$ (see Lemmas 6 and 7 below). We call such $V(t)$ a linear family of subspaces.

\section{Curves of vertical subspaces}

The principal objects we consider are curves of vertical subspaces along horizontal geodesics in $M^{n}$. We define them as follows: For every point $q$ in the space $M^{n}$ of the riemannian submersion $\pi: M^{n} \rightarrow B^{n-k}$ and every geodesic $\gamma$ issuing from the point $p=\pi(q)$ in the base $B^{n-k}$ of this submersion there exists a unique horizontal lift of $\gamma$, i.e., a geodesic $l$ issuing from $q$ such that $\pi(l(t))=\gamma(t)$, where $t$ is a natural parameter both on $l$ and $\gamma$, see [O'N2]. Such geodesics are called horizontal ones. In every point $l(t)$ the horizontal $\mathcal{H}_{l(t)}$ and vertical subspaces $\mathcal{V}_{l(t)}$ of a submersion $\pi$ are defined. Denote by $G_{n}^{k}$ the Grassmanian of all $k$-dimensional subspaces of the euclidean $n$-dimensional space $T_{q} M^{n}$ which we denote below by $R^{n}$. Then the curve $V_{l}(t)$ of vertical subspaces along $l$ is the curve in $G_{n}^{k}$ defined as follows:

$$
V_{l}(t)=I_{t}^{-1}\left(\mathcal{V}_{l(t)}\right)
$$

where $I_{t}$ is the parallel transport from $q$ to $l(t)$ along $l$. The obtained $V_{l}(t)$ is a family of vector $k$-subspaces of a fixed euclidean space $R^{n}$ (the tangent space $\left.T_{q} M^{n}\right)$. Generally, only the first element of this family $V_{l}(0)$ equals the vertical subspace $\mathcal{V}_{q}$. (If e.g., all $V_{l}(t)$ coincide with $\mathcal{V}_{q}$ and $M^{n}$ has nonnegative sectional curvature, then due to the splitting theorem, see [M2,M3], it follows that $M^{n}$ is locally a direct product.)

In this section we prove that norms of velocity vectors of curves $V_{l}$ of vertical subspaces are uniformly bounded, see Lemmas 4 and 5 below, and that these curves converge in $C^{1}$-norm to linear curves on longer and longer intervals as the curvature along $l$ tends to zero, when the point $q$ goes to infinity, see Lemma 6 .

First we estimate the velocity vector $\dot{V}_{l}(t)$ of the curve $V_{l}(t)$ through normal curvatures of the fiber $W_{p}$. By the normal curvature of the fiber $W_{p}$ at the point $q$ in direction $\eta$ tangent to $W_{p}$ and corresponding to the unit normal $e$, where the 
vector $e$ is horizontal (i.e., normal to $W_{p}$ ) we denote

$$
K_{\text {norm }}(\eta, e)=\left(\frac{D}{\partial s} e(s)_{\mid s=0}, \eta\right)=\left(\mathcal{V} \frac{D}{\partial(\mathcal{V} \eta)} \mathcal{H} e(s), \mathcal{V} \eta\right)
$$

where $q(s)$ be some curve in the fiber $W_{p}$ issuing from $q$ with velocity vector $\eta$ and $e(s)$ some unit horizontal vector field along $q(s)$ such that $e(0)=e$. Note that this definition is common, e.g., it equals

$$
\left(T_{\eta} e, \eta\right)=\left(\mathcal{V} \frac{D}{\partial(\mathcal{V} \eta)} \mathcal{H} e, \mathcal{V} \eta\right)
$$

where $T$ is O'Neill's tensor on pairs of vertical and horizontal vectors, see [O'N1]. Because the second fundamental form of the fiber $W_{p}$ corresponding to the normal $e$

$$
I I\left(\eta^{\prime}, \eta^{\prime \prime}, e\right)=\left(\mathcal{V} \frac{D}{\partial\left(\mathcal{V} \eta^{\prime}\right)} \mathcal{H} e, \mathcal{V} \eta^{\prime \prime}\right)
$$

is a symmetric one, the estimate on normal curvatures leads to an estimate on the norm of the second form:

$$
\|I I\|=\sup \left\{\left|I I\left(\eta^{\prime}, \eta^{\prime}, e\right)\right|\left\|\eta^{\prime}\right\|,\left\|\eta^{\prime}\right\|,\|e\| \equiv 1\right\}=\sup \left\{\left|K_{\text {norm }}(\eta, e)\right| \mid\|\eta\|,\|e\| \equiv 1\right\}
$$

In the next lemma we prove that the compactness ${ }^{1}$ of the base $B^{n-k}$ yields uniform boundedness of normal curvatures of fibers and due to (4.1) their second fundamental forms.

Lemma 4. Let $q(s),-s^{\prime}<s<s^{\prime}$ be some curve in the fiber $W_{p}$ such that $q=q(0)$, $e(s)$ be some horizontal unit vector field along $q(s)$ and $l(t)=\exp _{q}(t e(0))$. Then for some constant $\Lambda_{4}$, which depends only of the injectivity radius $r_{\text {inj }}(B)$ of a base $B$ and the curvature of a space $M^{n}$ of a submersion $\pi$ along $l$ the following is true

$$
\sup \left\{\left|K_{\text {norm }}(\eta, e)\right| \mid\|\eta\|,\|e\| \equiv 1\right\} \leq \Lambda_{4}
$$

Proof. Because every riemannian submersion does not increase distances, the following general statement is true: let $q_{1}$ and $q_{2}$ be two points in $M^{n}$ such that the distance $r$ between them equals the distance between their images $p_{1}$ and $p_{2}$ correspondingly under the submersion $\pi: M^{n} \rightarrow B^{n-k}$. Then all points from the fiber over $p_{1}$ have distance not less than $r$ to $q_{2}$, i.e., the fiber $W_{p_{1}}$ lies outside the metric ball $B\left(q_{2}, r\right)$ with the center $q_{2}$ and radius $r$ (and an easy geometric arguments show then that the normal curvatures of the fiber corresponding to the normal $\overline{q_{1} q_{2}}$ are less or equal to the corresponding curvatures of the ball $B\left(q_{2}, r\right)$

1 In fact, we need only to assume that $r_{i n j}\left(B^{n-k}\right)>0$. 
at the point $q_{1}$ ). Thus our estimate on normal curvatures is the corollary of a second variation formula due to the following arguments.

Denote by $q^{\prime}=l\left(r^{\prime}\right)$ for $r^{\prime}=r_{i n j}\left(B^{n-k}\right) / 3, q "=l(r ")$ for $r "=2 r_{i n j}\left(B^{n-k}\right) / 3$, and by $l^{\prime}=l(t), 0 \leq t \leq r^{\prime}$ and $l^{\prime \prime}=l(t), r^{\prime} \leq t \leq r$ " two intervals of $l$ connecting $q^{\prime}$ with $q$ and $q$ " with $q^{\prime}$ correspondingly. First we note that both $l^{\prime}$ and $l^{\prime}$ are minimal geodesics. Indeed, their images under submersion $\pi$ are geodesics in $B^{n-k}$ of length less than injectivity radius $r_{i n j}\left(B^{n-k}\right)$, i.e., are minimal geodesics. Because $l^{\prime}, l^{\prime \prime}$ are horizontal their length equal to the corresponding distances in $B^{n-k}$ between images $p^{\prime}=\pi\left(q^{\prime}\right)$ and $p=\pi(q)$ and $p "=\pi(q ")$ and $p^{\prime}=\pi\left(q^{\prime}\right)$ correspondingly, i.e., equal to $r^{\prime}$. As was said above, $\pi$ does not increase distances, so we see that length of $l^{\prime}$ and $l$ " are not bigger than distances between $q^{\prime}$ and $q$ and $q$ " and $q^{\prime}$ correspondingly, which means that $l^{\prime}, l^{\prime \prime}$ are minimal. In the same way we conclude that the geodesic $l=l(t), 0 \leq t \leq 2 r^{\prime}<r_{i n j}\left(B^{n-k}\right)$ connecting $q$ " and $q$ is also minimal. ${ }^{2}$

Now we take arbitrarily a family of minimal geodesics $l_{s}(t)$ connecting the point $q^{\prime}$ with $q(s)$. Then $l_{s}$ tends to the interval $l(t), 0 \leq t \leq r^{\prime}$ of $l^{\prime}$ when $s \rightarrow 0$. Indeed, if we suppose on the contrary the existence of some converging subsequence $l_{s_{j}}$ tending to some minimal geodesic $\tilde{l}^{\prime}$ connecting points $q^{\prime}$ and $q$ and different from $l^{\prime}$, then $\tilde{l}^{\prime}$ would be minimal, and $l$ " $\cup \tilde{l}^{\prime}$ would be a broken-geodesic path connecting $q "$ and $q$ and having length $2 r^{\prime}$ which equals the distance between $q$ " and $q$. But the path $l^{\prime} \cup \tilde{l^{\prime}}$ being broken at the point $q^{\prime}$ could be shortened, implying that $\operatorname{dist}(q ", q)<2 r^{\prime}$ and $l$ is not minimal. This contradiction yields that $\tilde{l}^{\prime}=l^{\prime}$, or that $l_{s}$ is a family of geodesics tending to $l^{\prime}$ as $s \rightarrow 0$. Therefore, $l_{s}$ defines a Jacobi vector field on $l^{\prime}$ :

$$
\mu(t)={\frac{\partial l_{s}(t)}{\partial s}}_{\mid s=0}, \quad 0 \leq t \leq r^{\prime}
$$

By definition $\mu(0)=\dot{q}(0)=\eta$ and we are assuming that $\eta$ is a unit vector, $\|\mu(0)\|=1$. If $L(s)$ denotes the length of $l_{s}(t), 0 \leq t \leq r^{\prime}$, then from arguments above we see that $L(s)$ attains a minimum at $s=0$. Hence, due to the second variation formula

$$
0 \leq L "(s)_{\mid s=0}=K_{n o r m}(\eta, e)+\int_{0}^{r^{\prime}}\left(\left\|\frac{D}{\partial t} \mu(t)\right\|^{2}-\left(R_{M}(\mu(t), i(t)) \dot{l}(t), \mu(t)\right)\right) d t
$$

where $R_{M}$ denotes a curvature tensor of $M^{n}$. Changing if necessary $e$ to $-e$ we assume that $K_{\text {norm }}(\eta, e) \leq 0$. Therefore, the claim of the lemma will follow if we prove that the integral part of (4.2) is uniformly bounded from above. By standard compactness arguments this is true for all points $q$ from an arbitrary fixed compact in $M^{n}$. If the point $q$ tends to infinity (this is exactly the case which we shall need and consider below, see the proof of Theorem A), then the sectional curvature of $M^{n}$ tends to zero due to our condition, and uniform boundedness is also clear.

\footnotetext{
2 Here we repeated some arguments from [M4].
} 
Indeed, consider $q$ such that $\operatorname{dist}(o, q)>\rho_{0}$ where $\rho_{0}$ is such that for all $\rho>\rho_{0}$ we have $\varkappa(\rho)<\varkappa_{0}=\left(\pi / 2 r^{\prime}\right)^{2}$, i.e., the sectional curvature of $M^{n}$ along $l^{\prime}$ by an absolute value is not bigger than $\varkappa_{0}$. Because of our choice and due to the Rauch comparison theorem (see [CE, Rauch I and Rauch II theorems 1.28 and 1.29]) we see that the Jacobi field $\mu(t), 0 \leq t \leq r^{\prime}$ has no conjugate points. Applying the same theorem to the field $\nu(t)=\mu\left(r^{\prime}-t\right), 0 \leq t \leq r^{\prime}$ such that $\nu(0)=0$ we deduce also that

$$
D \sin \left(\sqrt{\varkappa_{0}} t\right) \leq\|\nu(t)\| \leq D \sinh \left(\sqrt{\varkappa_{0}} t\right)
$$

where $D=\|(D \nu / \partial t)(0)\|$. Because $\left\|\nu\left(r^{\prime}\right)\right\|=1$ we find from (4.3) that $D \leq 1$ and

$$
\|\nu(t)\| \leq \sinh \left(\frac{\pi}{2}\right)
$$

Note also that our condition on the sectional curvature

$$
-\varkappa\|v \wedge w\|^{2} \leq\left(R_{M}(v, i) \dot{l}, v\right) \leq \varkappa\|v \wedge w\|^{2}
$$

implies that all eigenvalues of the symmetric bilinear form $\{v, w\} \rightarrow\left(R_{M}(v, i) i, w\right)$ belongs to an interval $[-\varkappa, \varkappa]$ so that for an arbitrary vector $v$ we have

$$
\left\|R_{M}(v, i) i\right\| \leq \varkappa\|v\| .
$$

Hence, from the Jacobi equation

$$
\frac{D^{2}}{\partial t^{2}} \nu(t)+R_{M}(\nu(t), i(t)) \dot{l}(t)=0
$$

it is not difficult to deduce

$$
\left\|\frac{D}{\partial t} \nu(t)\right\| \leq 1+\frac{\pi^{2}}{4 r^{\prime}} \sinh (\pi / 2)
$$

yielding a uniform estimate on the integral part of (4.2) and completing the proof of Lemma. Lemma 4 is proved.

Due to (4.1) the last Lemma gives also a uniform estimate of second fundamental forms of fibers of our submersion $\pi: M^{n} \rightarrow B^{n-k}$ implying in particular the following result.

Lemma 5. Let $q(s)$ be some curve in the fibre $W_{p}$ and $e(s)$ be a unit horizontal vector field along $q(s)$ obtained by lifting the same vector $\bar{e}$ from the base, i.e., $\pi_{*}(e(s)) \equiv \pi_{*}(e(0))$. Then for some constant $\Lambda_{5}$ depending only on $r_{i n j}\left(B^{n-k}\right)$ the following is true

$$
\left\|\frac{D}{\partial s} e(s)\right\| \leq \Lambda_{5}\|\dot{q}(s)\| .
$$


Proof. For $r=r_{i n j}(B)$ consider a family of horizontal geodesics $l_{s}(t)=\exp _{q(s)} t e(s)$, $0 \leq t \leq r$ issuing from $q(s)$ in directions $e(s)$. By definition of the horizontal field $e(s)$, all $l_{s}$ have the same image under the submersion $\pi: M^{n} \rightarrow B^{n-k}$, which is a minimal geodesic $\gamma(t)=\exp _{p} t \bar{e}, 0 \leq t \leq r$ on $B^{n-k}$. Thus the length $L(s)$ of all $l_{s}$ are equal, $L(s) \equiv r$. According to the second variation formula:

$$
\begin{aligned}
L^{\prime \prime}(s)_{\mid s=0} & =K_{\text {norm }}(\eta(r), \dot{l}(r))-K_{\text {norm }}(\eta(0), \dot{l}(0)) \\
& +\int_{0}^{r}\left(\left\|\frac{D}{\partial t} \eta(t)\right\|^{2}+\left(R_{M}(\eta(t), \dot{l}(t)) \dot{l}(t), \eta(t)\right)\right) d t,
\end{aligned}
$$

where $\eta(t)=\frac{\partial}{\partial t} l_{s}(t)_{\mid s=0}$ is the variation Jacobi field along $l(t)=l_{0}(t)$, and by $K_{\text {norm }}(\eta(t), i(t))$ we denote the normal curvature of the fiber $W_{\gamma(t)}$ in direction $\eta(t)$ according to the normal $i(t)$. Because of

$$
\frac{D}{\partial s} e(s)_{\mid s=0}=\frac{D}{\partial s} \frac{\partial}{\partial t} l_{s}(t)_{\mid s, t=0}=\frac{D}{\partial t} \frac{\partial}{\partial s} l_{s}(t)_{\mid s, t=0}=\frac{D}{\partial t} \eta(t)_{\mid t=0}
$$

the claim of the Lemma is equivalent to the estimate

$$
\left\|\frac{D}{\partial t} \eta(t)_{\mid t=0}\right\| \leq \Lambda_{5}\|\eta(0)\|,
$$

which we are deducing in a same way as above in Lemma 4 from the second variation formula (5.2), Lemma 4 uniform estimate on normal curvatures and Rauch comparison theorem. Clearly, we can assume $\|\eta(0)\|=1$. Due to Lemma 4 we have $\left|K_{\text {norm }}(\eta(t), \dot{l}(t))\right| \leq \Lambda_{4}\|\eta(t)\|$, and again by standard compactness arguments (5.3) follows for an arbitrary $q$ from every fixed compact domain in $M^{n}$. If the point $q$ tends to infinity (which is again the only what we need below, see the proof of Theorem A), then the sectional curvature of $M^{n}$ tends to zero due to our condition on $M^{n}$. For $q$ with $\operatorname{dist}(o, q)>\rho_{0}$, where $\rho_{0}$ is such that for all $\rho>\rho_{0}$ we have $\varkappa(\rho)<\varkappa_{0}=(\pi / 2 r)^{2}$, the sectional curvature of $M^{n}$ along $l$ by an absolute value is not bigger than $\varkappa_{0}$. Now we decompose the Jacobi field $\eta(t)=\mu(t)+\nu(t)$ into two Jacobi fields such that $\mu(0)=\eta(0),(D \mu / \partial t)(0)=0$ and $\mu(0)=0,(D \nu / \partial t)(0)=(D \eta / \partial t)(0)$. Because of our choice of $\varkappa_{0}$ the Jacobi fields $\mu(t)$ has no focal points and $\nu(t)$ has no conjugate points for $0 \leq t \leq r$. Therefore, by the Rauch comparison theorem (see [CE, Rauch I and Rauch II theorems 1.28 and 1.29]) we obtain the following estimates:

$$
\cos \left(\sqrt{\varkappa_{0}} t\right) \leq\|\mu(t)\| \leq \cosh \left(\sqrt{\varkappa_{0}} t\right)
$$

and

$$
D \sin \left(\sqrt{\varkappa_{0}} t\right) \leq\|\nu(t)\| \leq D \sinh \left(\sqrt{\varkappa_{0}} t\right)
$$

where $D=\|(D \eta / \partial t)(0)\|$ is the number we are estimating. These gives us for $0 \leq t \leq r$

$$
\|\eta(t)\| \leq D \sinh (r)+B
$$


where $B$ equals $\cosh (\pi / 2)$. Thus from the Jacobi equation

$$
\frac{D^{2}}{\partial t^{2}} \eta(t)+R_{M}(\eta(t), i(t)) \dot{l}(t)=0
$$

with the help of (4.6) it is not difficult to deduce

$$
\left\|\frac{D}{\partial t} \eta(t)\right\|-\left\|\frac{D}{\partial t} \eta(0)\right\| \mid \leq \varkappa_{0} r(D \sinh (r)+B)
$$

which under the conditions

$$
\varkappa_{0} \sinh (r) \leq 1 \quad \text { and } \quad \varkappa_{0} B \leq 1
$$

leads to

$$
\left|\left\|\frac{D}{\partial t} \eta(t)\right\|-D\right| \leq r D+r, \quad \text { and } \quad\left|\left\|\frac{D}{\partial t} \eta(t)\right\|\right| \leq(1+r) D+r,
$$

and

$$
\left|r\left\|\frac{D}{\partial t} \eta(0)\right\|^{2}-\int_{0}^{r}\left\|\frac{D}{\partial t} \eta(t)\right\|^{2} d t\right| \leq r^{2}(2+r) D^{2}+2 r^{2}(1+r) D+r^{3} .
$$

Due to (5.2) the last inequality gives us

$$
r D^{2} \leq r^{2}(2+r) D^{2}+2 r^{2}(1+r) D+r^{3}+\Lambda_{4}(1+(D r+B)) .
$$

Thus, finding some $\rho_{1}>\rho_{0}$ such that for $\rho>\rho_{1}$ we have $\varkappa(\rho)<\sinh ^{-1}(r), B^{-1}$ to satisfy (5.7) and finally obtain

$$
D \leq \Lambda_{5}
$$

for some constant $\Lambda_{5}$ depending only on $r=r_{i n j}(B)$ proving the claim of the Lemma. Lemma 5 is proved.

Remark 4. Note that in Lemmas 4 and 5 we do not have to assume that fibers of our submersion are smooth submanifolds: the corresponding definitions of normal curvatures and second fundamental form could be given in a barrier sense, see for instance [C] or [M5]. Our Lemmas 4, 5 in this case prove that fibers have uniformly bounded normal curvatures in barrier sense (Lemma 4), and that derivatives of Jacobi fields generated by horizontal variations of horizontal geodesics are also bounded (Lemma 5). In particular, Lemma 5 estimate is true in a case of open manifolds of nonnegative sectional curvature, where it was proved only that the corresponding riemannian submersion is of $C^{1,1}$-class (e.g., due to our arguments 
in Lemmas 4 and 5). This allows us to apply Theorem A for open manifolds of nonnegative curvature proving topological gap Theorem B.

Now we take arbitrarily some point $p$ and a geodesic $\gamma(t)=\exp _{p}(t \bar{e})$ in a base $B^{n-k}$, and consider their horizontal lifts in $M^{n}$, i.e., a point $q$ of a fiber $W_{p}$ over $p$ and a horizontal geodesic $l_{q}(t)=\exp _{q}(t e)$. In the next lemma we prove that when $q$ goes to infinity the curve of vertical subspaces $V_{l_{q}}$ tends in $C^{1}$-norm to some linear family of subspaces.

Lemma 6. For $T$ arbitrary big and $\epsilon$ arbitrary small there exists $\rho(\epsilon, T)$ such that for all $q$ with $\operatorname{dist}(o, q)>\rho(\epsilon, T)$ the curve of vertical subspaces $V_{l_{q}}(t), 0 \leq t \leq T$ along $l_{q}$ is $\epsilon$ close in $C^{1}$-norm to some linear family $\bar{V}(t), 0 \leq t \leq T$.

Proof. Let $q_{i}(s), i=1, \ldots, k$ be some curves in the fiber $W_{p}$ issuing from the point $q$ in directions of vectors $\eta_{i}$ which form a base of a vertical space $\mathcal{V}_{q}$. Denote by $l_{q, s, i}(t)$ horizontal lifts of $\gamma$ to $q_{i}(s)$, i.e., such that

$$
\pi\left(l_{q, s, i}(t)\right) \equiv \gamma(t)
$$

Because of (6.1) variation fields

$$
\eta_{i}(t)=\frac{\partial}{\partial s} l_{q, s, i}(t)_{\mid s=0}
$$

are vertical ones and such that for any given $t$ vectors $\left\{\eta_{i}(t), i=1, \ldots, k\right\}$ generate $\mathcal{V}_{l_{q}(t)}$. By definition the curve of vertical subspaces $V_{l}(t)=I_{t}^{-1} \mathcal{V}_{l_{q}}$ is generated by parallel transports $e_{i}(t)$ of these vectors $\left\{\eta_{i}(t), i=1, \ldots, k\right\}$ along $l_{q}$ to the point $q$, i.e.,

$$
V(t)=<e_{i}(t), i=1, \ldots, k>\quad \text { where } \quad e_{i}(t)=I_{t}^{-1}\left(\eta_{i}(t)\right),
$$

and $I_{t}^{-1}$ is a parallel transport along geodesic $l_{q}$ from the point $l_{q}(t)$ to $q$. To simplify notations, we denote our curve $V_{l_{q}}(t)$ by $V(t)$ and $l_{q}$ by $l$. Clearly

$$
e_{i}^{\prime}(t)=I_{t}^{-1}\left(\frac{D}{\partial t} \eta_{i}(t)\right) \quad \text { and } \quad e^{n_{i}}(t)=I_{t}^{-1}\left(\frac{D^{2}}{\partial t^{2}} \eta_{i}(t)\right)
$$

and from the Jacobi equation (5.6) we see that vector fields $e_{i}(t)$ in the euclidean space $R^{n}=T_{q} M^{n}$ are solutions of the corresponding equation

$$
e_{i}{ }^{\prime \prime}(t)+R_{t}\left(e_{i}(t)\right)=0
$$

where the linear self-adjoint operator $R_{t}$ is defined by $R_{t}(v)=I_{t}^{-1} R_{M}\left(I_{t}(v), \dot{l}(t)\right)$ $i(t)$. As above due to (4.6)

$$
\left\|R_{t}\right\| \leq \varkappa\left(\rho_{l}\right)
$$


where $\rho_{l}=\inf \{\operatorname{dist}(o, l(t)) \mid 0 \leq t \leq T\}$. From the triangle inequality easy to see that $\rho_{l} \geq \rho-T$, where by $\rho$ we denote $\operatorname{dist}(o, q)$. Because $\varkappa(\rho) \rightarrow 0$ as $\rho \rightarrow \infty$ we are able to prove that our curve $V(t)$ tends to the linear family $\bar{V}(t)$ generated by

$$
\bar{e}_{i}(t)=e_{i}(0)+t e_{i}^{\prime}(0)
$$

Here, as above in Remark 3, we have to repeat that for some $t_{j}, j=1, \ldots, m, m \leq n$ a dimension of $\bar{V}(t)$ could be less than $k$, so that only open intervals $\bar{V}(t), t_{j}<t<$ $t_{j+1}$ of $\bar{V}(t)$ are linear curves. Thus we consider below $t \neq t_{j}$ and verify that our curve $V(t)$ converges on intervals $\left(t_{j}, t_{j+1}\right)$ to linear curves $\bar{V}(t), t_{j}<t<t_{j+1}$.

Take some $t_{j}<t^{\prime}<t_{j+1}$ and find arbitrarily an orthonormal base $\left\{e_{i}\left(t^{\prime}\right), i=\right.$ $1, \ldots, k\}$ of $V\left(t^{\prime}\right)$. Without loss of generality we can assume that these vectors are values of Jacobi fields $\eta_{i}$ along $l$, i.e., $e_{i}\left(t^{\prime}\right)=I_{t}^{-1}\left(\eta_{i}\left(t^{\prime}\right)\right)$. Compare $e_{i}\left(t^{\prime}\right)$ with $\bar{e}_{i}\left(t^{\prime}\right)$, where $\bar{e}_{i}(t)=\eta_{i}(0)+t D \eta_{i}(0)$ is the vector from $\bar{V}\left(t^{\prime}\right)$. The claim of the lemma will follow if we prove that $e\left(t^{\prime}\right)$ and $\bar{e}\left(t^{\prime}\right)$ and their derivatives $e^{\prime}\left(t^{\prime}\right)$ and $\bar{e}^{\prime}\left(t^{\prime}\right)$ are $\epsilon$-close.

First, let us show that the norm of every $\eta_{i}(t)$ is bounded by some function depending only on $\varkappa\left(\rho_{l}\right)$ and $T$. Indeed, by definition $\left\|\eta_{i}\left(t^{\prime}\right)\right\|=1$, so by Lemma 5 and due to (5.2) we have $\left\|D \eta_{i}\left(t^{\prime}\right)\right\| \leq \Lambda_{5}$ for some constant $\Lambda_{5}$. Therefore, representing as above in Lemma 5 the field $\eta_{i}(t)$ as a sum of two Jacobi fields $\nu_{i}(t)$ and $\mu_{i}(t)$ such that $\left\|D \nu_{i}\left(t^{\prime}\right)\right\|=0$ and $\left\|\mu_{i}\left(t^{\prime}\right)\right\|=0$ by the Rauch's Comparison Theorem (comparing $M^{n}$ with the hyperbolic plane of constant curvature $\varkappa$, see (5.4) and (5.5) above) we see that

$$
\sqrt{\varkappa\left(\rho_{l}\right) T} \leq \pi / 2
$$

implies for the Jacobi fields the following: $\mu_{i}$ has no focal points, and $\nu_{i}$ has no conjugate points for $0 \leq t \leq T$, and the following estimates are true:

$$
\left\|\mu_{i}(t)\right\| \leq \cosh \left(\sqrt{\varkappa\left(\rho_{l}\right)} t\right)
$$

and

$$
\left\|\nu_{i}(t)\right\| \leq \Lambda_{5} \sinh \left(\sqrt{\varkappa\left(\rho_{l}\right)} t\right)
$$

Because $\left\|e_{i}(t)\right\| \equiv\left\|\eta_{i}(t)\right\|$ the last two inequalities give

$$
\left\|e_{i}(t)\right\| \leq \cosh \left(\sqrt{\varkappa\left(\rho_{l}\right)} t\right)+\Lambda_{5} \sinh \left(\sqrt{\varkappa\left(\rho_{l}\right)} t\right) .
$$

Thus from (6.3), (6.4) and (6.6) we easily have

$$
\left\|e_{i}(t)-\left(e_{i}(0)+t e_{i}^{\prime}(t)\right)\right\| \leq \Lambda_{6} \varkappa\left(\rho_{l}\right) T^{2}
$$

and

$$
\left\|e_{i}^{\prime}(t)-e_{i}^{\prime}(t)\right\| \leq \Lambda_{6} \varkappa\left(\rho_{l}\right) T
$$


where

$$
\Lambda_{6}=\cosh (\pi / 2)+\Lambda_{5} \sinh (\pi / 2) .
$$

If in addition

$$
\varkappa\left(\rho_{l}\right) T^{2}<\epsilon \Lambda_{6}^{-1}
$$

we deduce

$$
\left\|e_{i}(t)-\bar{e}_{i}(t)\right\|<\epsilon \quad \text { and } \quad\left\|e_{i}^{\prime}(t)-\bar{e}_{i}^{\prime}(t)\right\|<\epsilon .
$$

As we already note, due to the triangle inequality $\rho_{l} \geq \rho-T$. Therefore, to complete the proof note that to satisfy (6.6) and (6.12) it is sufficient for given $\epsilon$ and $T$ choose first some $0<\varkappa<\epsilon T^{-2}$ and $0<\varkappa<\epsilon T^{-2} \Lambda_{6}^{-1}$, and then find $\rho_{0}$ such that $\varkappa(\rho)<\varkappa$ for all $\rho>\rho_{0}$. Then estimates of the lemma (6.13) will be satisfied for all points $q$ such that $\rho(q)>\rho_{0}+T$.

We end this section with a statement similar to the main result of the section 1: proving that the length of a curve of vertical subspaces $V_{l}(t), 0 \leq t \leq T$ stays bounded when $T \rightarrow \infty$ for some suitable choice of $\epsilon$ and corresponding $\rho(\epsilon, T) \rightarrow$ $\infty$.

Lemma 7. Let $\epsilon=T^{-2}$ and $\rho=\rho(\epsilon, T)$ be a number defined in the previous Lemma 6. Then

$$
\int_{0}^{T}\left\|\dot{V}_{l}(t)\right\| d t \quad, \quad \int_{0}^{T}\left\|\dot{V}_{l}(t)\right\|^{2} d t \leq \Lambda_{7}
$$

for some uniform constant $\Lambda_{7}$ and every horizontal geodesic $l$ issuing from the point $q$, if only $\epsilon$ is sufficiently small and $\operatorname{dist}(o, q)>\rho(\epsilon, T)$.

Proof. To prove the claim of the lemma we compare the length of the curve of vertical subspaces $V_{l}(t), 0 \leq t \leq T$ with the length of the corresponding $\bar{V}(t), 0 \leq$ $t \leq T$ constructed in the proof of Lemma 6 . Here again (see Remark 3 and Lemma 6 above) we consider only $t \neq t_{j}$ such that intervals $\bar{V}(t), t_{j}<t<t_{j+1}$ of $\bar{V}(t)$ are linear curves, and verify that the length of our curve $V_{l}(t)$ converges to the sum of length of linear curves $\bar{V}(t), t_{j}<t<t_{j+1}$.

Divide the interval $[0, T]$ into two subsets $I_{1}=\left\{t \mid\left\|\dot{V}_{l}(t)\right\|<T^{-1}\right\}$ and $I_{2}=$ $\left\{t \mid\left\|\dot{V}_{l}(t)\right\| \geq T^{-1}\right\}$. Obviously,

$$
\int_{I_{1}}\left\|\dot{V}_{l}(t)\right\| d t \quad, \quad \int_{I_{1}}\left\|\dot{V}_{l}(t)\right\|^{2} d t \leq \frac{1}{T}
$$

To estimate the length of $\left\{V_{l}(t), t \in I_{2}\right\}$ we note that due to Lemma $6 \dot{V}_{l}(t)$ and $\bar{V}(t)$ are $\epsilon$-close where $\epsilon=T^{-2}$ an inequality $\left\|\dot{V}_{l}(t)\right\| \geq T^{-1}$ means that directions of the vectors $\dot{V}_{l}(t)$ and $\bar{V}(t)$ are $T^{-1}$-close:

$$
\angle\left(\dot{V}_{l}(t), \bar{V}(t)\right)<T^{-1}
$$


Because of this the length of $V_{l}(t), t \in I_{2}$ is close to the length of $\bar{V}(t), t \in I_{2}$ by the following standard arguments. Consider sufficiently small $r$-tubular neighborhood $U$ of $\bar{V}(t)$ in $G_{n}^{k}$ for some $r<r_{i n j}\left(G_{n}^{k}\right)$ and such that 1) the projection $p r: U \rightarrow$ $\bar{V}(t)$ sending a point $V$ to the nearest to it point on the curve $\bar{V}(t)$ is well defined in this neighborhood, and 2) has bounded differential. The existence of such $r$ follows from the uniform boundedness of the geodesic curvature of $\bar{V}(t)$, see Lemma 2 . Then every curve $V_{l}(t)$ in $U$ having directions $\dot{V}_{l}(t)$ sufficiently close to $\bar{V}(t)$ will be transversal to the fibers of $p r$. Thus we conclude that

a) for $r$ and $\epsilon$ sufficiently small the map $p r:\left\{V_{l}(t), t \in I_{2}\right\} \rightarrow\{\bar{V}(t), t \in[0, T]\}$ is injective to its image, and

b) the length of $\left\{V_{l}(t), t \in I_{2}\right\}$ is bounded in terms of the length of its image $\operatorname{pr}\left(\left\{V_{l}(t), t \in I_{2}\right\}\right.$ ), the angle between $\bar{V}(t)$ and $\dot{V}(t)$ and $r$ (we omit this standard estimate. It involves first and second variation formulas, and could be found in almost all manuals on riemannian geometry).

Therefore, (7.2) for small $\epsilon$ yields

$$
\int_{I_{2}}\left\|\dot{V}_{l}(t)\right\| d t \leq \Lambda_{7}^{1} \int_{0}^{T}\|\bar{V}(t)\| d t \leq(k+1) \Lambda_{3} \Lambda_{7}^{1}
$$

for some uniform constant $\Lambda_{7}^{1}$ (where, of course, $\Lambda_{7} \rightarrow 1$ as $\epsilon \rightarrow 0$ ). Due to Lemma 5 we have $\left\|\dot{V}_{l}(t)\right\| \leq \Lambda_{5}$, hence the last inequality gives also

$$
\int_{I_{2}}\left\|\dot{V}_{l}(t)\right\|^{2} d t \leq(k+1) \Lambda_{3} \Lambda_{5} \Lambda_{7}^{1}
$$

which together with (7.1) completes the proof of the lemma. Lemma 7 is proved.

Now we are ready to prove Theorem A.

\section{Proof of Theorem A}

Let $\pi: M^{n} \rightarrow B^{n-k}$ be a Riemannian submersion with $M^{n}$ flat at infinity, and $p$ be some point in $B^{n-k}, \bar{X}$ and $\bar{Y}$ some unit vectors of $T_{p} B^{n-k}$ and $q$ be some point in $M^{n}$ such that $\pi(q)=p$, and $X, Y$ be horizontal lifts of $\bar{X}$ and $\bar{Y}$ in $q$, i.e., horizontal unit vectors from $T_{q} M^{n}$ such that $\pi_{*}(X)=\bar{X}$ and $\pi_{*}(Y)=\bar{Y}$. Then, according to [O'N1] for the sectional curvatures $K_{M}$ of $M^{n}$ and $\bar{K}_{B}$ of $B^{n-k}$ in two-dimensional directions $\{X, Y\}$ and $\{\bar{X}, \bar{Y}\}$ correspondingly, the following is true:

$$
\bar{K}_{B}(p, \bar{X}, \bar{Y})=K_{M}(q, X, Y)+3\left\|A_{X}(Y)\right\|^{2},
$$

where $A_{X}(Y)$ is the O'Neill tensor of the horizontal distribution equals $\mathcal{V} \nabla_{X}(Y)$. Let $\eta$ be a vertical unit vector at $q$ parallel to $A_{X}(Y)$. Then if $\eta(t)$ is the Jacobi vertical vector field with $\eta(0)=\eta$ as before along the horizontal geodesic $l(t)$ 
issuing from the point $q$ in direction $X$, and $Y(t)$ - any horizontal field along $l$ such that $Y(0)=Y$, then from $(\eta(t), Y(t)) \equiv 0$ we have

$$
\left\|A_{X}(Y)\right\|=\left|\left(\eta, A_{X}(Y)\right)\right|=|(Y, D \eta(0))|=|(Y, \mathcal{H} D \eta(0))| \leq\|\mathcal{H} D\|=\left\|\dot{V}_{l}(0)\right\|,
$$

see Lemma 1 equality.

Because $M^{n}$ is open, it is easy to see, that all fibers $W_{p}$ are unbounded (otherwise $M^{n}$ would be compact), and for every point $p$ of $B^{n-k}$ and $\rho$ there exists $q$ from $W_{p}$ with $\rho(o, q)>\rho$. Chose $\varkappa>0$ arbitrary small and let $\rho^{\prime}$ is the number such that $k(\rho)<\varkappa$ for all $\rho>\rho^{\prime}$. For arbitrary $T$ find another $\rho^{\prime \prime}$ according to Lemma 7. Then from O'Neill formula (1), estimate (2), Lemma 7 estimates and $\left|K_{M}(l(t), \dot{l}(t), Y(t))\right| \leq \varkappa$ we have

$$
\frac{1}{T} \int_{0}^{T}\left|\bar{K}_{B}(\gamma(t), \dot{\gamma}(t), \bar{Y}(t))\right| d t \leq \varkappa+\frac{3}{T} \int_{0}^{T}\left\|\dot{V}_{l}(t)\right\|^{2} d t \leq \varkappa+\frac{3}{T} \Lambda_{7},
$$

for $\rho>\rho^{\prime}, \rho "$. Consider a map $\phi_{t}$ sending $(p, \bar{X}, \bar{Y})$ to $(\gamma(t), \dot{\gamma}(t), \bar{Y}(t))$. Like a geodesic flow, i.e., the map sending $(p, \bar{X})$ to $(\gamma(t), \dot{\gamma}(t))$; this map preserves the volume form of the bundle $S^{2} B^{n-k}$ of the pairs of unit normal vectors in $B^{n-k}$. Therefore, according to the Birkhoff-Khintchine theorem the left hand side of (3) under the constraint $T \rightarrow \infty$ tends almost everywhere to the mean value function $\bar{K}^{*}$ of the function $\left|\bar{K}_{B}(p, \bar{X}, \bar{Y})\right|$ on $S^{2} B^{n-k}$ and equals zero according to (3). Thus the integral of $\bar{K}^{*}$ over $S^{2} B^{n-k}$ equals zero. According to the same Birkhoff-Khintchine theorem this integral of $\bar{K}^{*}$ over $S^{2} B^{n-k}$ equals the integral of $\left|\bar{K}_{B}(p, \bar{X}, \bar{Y})\right|$ over $S^{2} B^{n-k}$, implying that $\bar{K}_{B}(p, \bar{X}, \bar{Y}) \equiv 0$, or $B^{n-k}$ is flat. Theorem A is proved.

\section{Mean normal curvatures of fibers}

We conclude this paper with a note, that Lemma 7 uniform estimate allows us to prove not only that the base $B^{n-k}$ is flat, but also that mean integral values of normal curvatures of fibers along any interval $l=\{l(t), 0 \geq t \geq T\}$ of length $T$ of an arbitrary horizontal geodesic in $M^{n}$ tend to zero as $T \rightarrow \infty$ and this interval $l$ tends to infinity. Namely, let $l(t)$ be some horizontal geodesic going through some arbitrary chosen point $q$ in a horizontal direction $e$ of $\mathcal{H}_{q}$. Consider the function $K_{\text {norm }}(\eta, e)$ - the normal curvature of the fiber containing the point $q$ in some vertical direction $\eta$ corresponding to the normal $e$ of this fiber. Denote by $N(t)$ the maximum of all $\left|K_{\text {norm }}(\eta(t), \dot{l}(t))\right|$ over all vertical vectors $\eta(t)$ at the point $l(t)$, and by $\rho_{l}=\inf \{\operatorname{dist}(o, l(t) \mid 0 \leq t \leq T\}$. Then the following is true:

\section{Lemma 8.}

$$
\frac{1}{T} \int_{0}^{T} N(t) d t<\Lambda_{8}\left(\frac{1}{\sqrt{T}}+\sqrt{\varkappa\left(\rho_{l}\right)}\right)
$$


for some universal constant $\Lambda_{8}$.

Proof. Let as before $\eta(t)$ be the Jacobi variation field along $l(t)$ generated by the family $l_{s}(t), l_{0}(t)=l(t)$ of horizontal geodesics, $\eta(t)=\frac{\partial}{\partial s} l_{s}(t)_{\mid s=0}$ and $e(s)=$ $i_{s}(0)$. Then $\frac{D}{\partial s} e(s)_{\mid s=0}=\frac{D}{\partial t} \eta(t)_{\mid t=0}$ and for $K(t)=K_{\text {norm }}(\eta(t), \dot{l}(t))$ by a direct calculation we have

$$
\begin{gathered}
K^{\prime}(t)=\left(\left(\frac{D}{\partial s} e(t), \eta(t)\right) /(\eta(t), \eta(t))\right)^{\prime}=\left(\left(\frac{D}{\partial t} \eta(t), \eta(t)\right) /(\eta(t), \eta(t))\right)^{\prime}= \\
\frac{\left(\frac{D \eta}{\partial s}(t), \frac{D \eta}{\partial t}(t)\right)(\eta(t), \eta(t))-2\left(\frac{D \eta}{\partial t}(t), \eta(t)\right)^{2}}{(\eta(t), \eta(t))^{2}}+\frac{\left(\frac{D^{2} \eta}{\partial t^{2}}(t), \eta(t)\right)}{(\eta(t), \eta(t))^{2}} .
\end{gathered}
$$

If we assume that at the moment $t=0$ our vector $\eta(0)$ is the eigenvector of the second fundamental form $\mathbf{I I}(0)$ of the fiber $W_{p}$ in the considered point $q$ corresponding to the normal $e=i(0)$, then the last formula and the Jacobi equation for $\eta(t)$ give:

$$
K^{\prime}(0)+K^{2}(0)=\frac{\left\|\mathcal{H} \frac{D \eta}{\partial t}(0)\right\|^{2}}{\|\eta(0)\|^{2}}+K_{M}(l(0), \dot{l}(0), \eta(0)) .
$$

Obviously, the same formula for the derivative of the normal curvature of the fiber is true for any $t$ if $\eta(t)$ is a unit eigenvector of the second form corresponding to the normal $i(t)$. For arbitrary $t$ taking in the last formula sum over all eigenvectors $\eta_{i}(t)$ of the second form $\mathbf{I I}(t)$ of the fiber $W_{\gamma(t)}$ (where $\left.\gamma(t)=\pi(l(t))\right)$ corresponding to the normal $e(t)=\dot{l}(t)$ we obtain the following formula for the trace $A_{1}(t)$ of the second form $\mathbf{I I}(t)$ of the fiber $W_{\gamma(t)}$ according to the normal $\dot{l}(t)$, see [M2], [M3]:

$$
A_{1}^{\prime}(t)+\left\|A_{2}(t)\right\|=\sum_{i=1}^{k} \frac{\left\|\mathcal{H} \frac{D \eta_{i}}{\partial t}(t)\right\|^{2}}{\left\|\eta_{i}(t)\right\|^{2}}+K_{M}^{k}(l(t), i(t)),
$$

where by $\left\|A_{2}(t)\right\|$ we denote the sum of squares of eigenvalues of the form $\mathbf{I I}(t)$ and by

$$
K_{M}^{k}(l(t), \dot{l}(t))=\sum_{i=1}^{k} K_{M}^{k}\left(l(t), \dot{l}(t), \eta_{i}(t)\right)
$$

the "partial (or vertical) Ricci curvature" in direction $i(t)$. According to Lemma 1

$$
\left\|\dot{V}_{l}(t)\right\|^{2}=\sum_{i=1}^{k} \frac{\left\|\mathcal{H} \frac{D \eta_{i}}{\partial t}(t)\right\|^{2}}{\left\|\eta_{i}(t)\right\|^{2}}
$$

which leads to the formula:

$$
A_{1}^{\prime}(t)+\left\|A_{2}(t)\right\|=\left\|\dot{V}_{l}(t)\right\|^{2}+K_{M}^{k}(l(t), i(t)) .
$$


From our condition on the curvature we have $\left|K_{M}^{k}(l(t), \dot{l}(t))\right| \leq k \varkappa\left(\rho_{l}\right)$. As follows from Lemma $5\left\|\dot{V}_{l}(t)\right\| \leq \Lambda_{5}$, hence

$$
A_{1}^{\prime}(t)+\left\|A_{2}(t)\right\| \leq \Lambda_{5}\left\|\dot{V}_{l}(t)\right\|+k \varkappa\left(\rho_{l}\right) .
$$

Taking the integral of the above inequality over an interval $[0, T]$ and using the estimate $\left|A_{1}(t)\right| \leq k \Lambda_{5}$ following from Lemma 5 we get:

$$
\frac{1}{T} \int_{0}^{T}\left\|A_{2}(t)\right\| d t \leq \frac{2 k \Lambda_{5}}{T}+\frac{\Lambda_{5}}{T} \int_{0}^{T}\left\|\dot{V}_{l}(t)\right\| d t+k \varkappa\left(\rho_{l}\right)
$$

which due to Lemma 7 gives us the following estimate:

$$
\frac{1}{T} \int_{0}^{T}\left\|A_{2}(t)\right\| d t \leq \frac{\Lambda_{8}^{\prime}}{T}+k \varkappa\left(\rho_{l}\right)
$$

for some uniform constant $\Lambda_{8}^{\prime}$. By definition $|N(t)| \leq \sqrt{\left\|A_{2}(t)\right\|}$, thus the claim of the lemma easily follows from Cauchi-Bunyakowsky inequality. Lemma 8 is proved.

\section{References}

[CE] J. Cheeger, D. Ebin, Comparison Theorems in Riemannian Geometry, Math. Library 9 North Holland, Amsterdam 1975.

[CG] J. Cheeger, D. Gromoll, On the structure of complete manifolds of nonnegative curvature, Ann. Math. 96(3) (1972), 413-443.

[EES] J.-H. Eschenburg, V. Schroeder, M. Strake, Curvature at infinity of open nonnegatively curved manifolds, J. Diff. Geom. 30 (1989), 155-166.

[GG] D. Gromoll, K. Grove, The low-dimensional metric foliations of euclidean spheres, $J$. Diff. Geom. 28 (1988), 143-156.

[KS] A. Kasue, K. Sugahara, Gap theorems for certain submanifolds of euclidean spaces and hyperbolic space forms, Osaka J. Math. 24 (1987), 679-704.

[M1] V. Marenich, The topological gap phenomenon for open manifolds of nonnegative curvature, Soviet Math. Dokl. 32 (1985), 440-443.

[M2] V. Marenich, The metric structure of open manifolds of nonnegative curvature, Soviet Math. Dokl. 24 (1981), 595-597. (complete version (in russian) under the same title in: Ukraine Geom. Sb. 26 (1983), 79-96.)

[M3] V. Marenich, The holonomy in open manifolds of nonnegative curvature, Mich. Math. J. 43(2) (1996), 263-272.

[M4] V. Marenich, Submersions of open manifolds of nonnegative curvature, Preprint RP 11/95 IMEEC - UNICAMP 1995.

[M5] V. Marenich, Structure of open manifolds of nonnegative curvature I, II, Siberian Advances in Mathematics 2(4) (1992), 104-146; 3(1) (1993), 129-151. 
[O'N1] B. O'Neill, The fundamental equations of submersion, Mich. Math. J. 13(4) (1966), 459-469.

[O'N2] B. O'Neill, Submersions and geodesics, Duke Math. J. 34 (1967), 363-373.

[P] G. Perelman, Proof of the soul conjecture of Cheeger and Gromoll, J. Diff. Geom. 40 (1994), 209-212.

[W ] G. Walschap, Metric foliations and curvature, J. Geom. Anal. 2 (1992), 373-381.

Valery Marenich

IMECC - UNICAMP

Caixa Postal 6065

CEP 13081-970

Campinas, Brazil

e-mail: marenich@ ime.unicamp.br

(Received: October 21, 1997; revised: June 2, 1998) 